Aus den hier mittgeteilten Daten ergibt sich folgendes:

Der Zug bez. Aufenthalt der Seidenschwänze währte vom 21. November 1920 bis 9. Mai 1921.

Erstes Erscheinen in der Schweiz 21. XI. 20, letztes in Niederösterreich 2. V., bezw. Mähren, 3 und 9. V.

Vermehrtes Auftreten im Jannar (Schweiz, Salzburg, Niederösterreich, Preufsen, Mähren).

Grölste Ausbreitung im Februar (Schweiz, Salzburg, Oberund Niederösterreich, Steiermark, Mähren, Bayern).

Abflauung des Zuges im März (Schweiz, Bayern, Niederösterreich, Mähren.)

Erlöschen des Zuges, Nachzügler im April (Bosnien, Mähren, Preufsen), im Mai in Niederösterreich und Mähren.

Am längsten hielten sich die Seidenschwänze in der Schweiz auf (November 1920 bis März 1921).

Sie erschienen in Scharen, Schwärmen (Schweiz, Oberösterreich, Kimitschthal? Mähren), häufiger in Flügen (Brandenburg, Bayern, Salzburg, Stejermark, Oberösterreich und Mähren) am öftesten in kleinen Gesellschaften.

Die meisten Beobachtungen der Zahl nach entfallen auf Oesterreich und die Cschecho-Slowakei. Deutschland hat das Erscheinen der Fremdlinge weniger in den Kreis seiner Beobachtungen gezogen, doch auch die dürftigen Daten von dort lassen ein mindestens gleich häufiges Auftreten wie in der Cschecho-Slowakei und Oesterreich vermuten und erwarten.

Nach den wertvollen Angaben Dr. Karásek's mufs die Zahl der in Mähren und wohl auch in Schlesien aufgetretenen Seidenschwänze eine ganz gewaltige gewesen sein und scheinen selbe hier ihr Verbreitungszentrum gefunden zu haben, da sonst nirgendsher von solchen Massen berichtet wurde. Es dürfte daher meine noch vor Empfang des Berichtes Dr. Karáseks geäufserte Ansicht, dafs der Anzug aus dem Osten erfolgte, durch diesen eine weitere Stütze erhalten, auch die späte erste Beobachtung - 21. November, Schweiz - deutet darauf hin, da bei den früberen grofsen Zügen die ersten Beobachtungen in den Oktober, fallen.

Tännenhof b. Hallein, September 1921.

\title{
Andreas Johannes Jäckel.
}

Am 6. Januar 1822 waren es 100 Jahre, dafs der Altmeister der bayerischen Zoologie, Pfarrer Andreas Johannes J ä ck el als Sohn des Kirchners zu St. Aegydien, Jakob J., in Nürnberg das Licht der Welt erblickte. Pietät und Dankbarkeit erheischen es, dieses um unsere Wisseuschaft so hochverdienten Mannes auch in einer ornithologischen Zeitschrift zu gedenken. 
J. verlebte eine an Entbehrungen reiche Jugend, besuchte als einziges von seinen Geschwistern das Melanchtbon-Gymnasium zu Nürnberg, studierte in Erlangen Theologie und hatte dann als Vikar die Pfarrstellen von Kloster Sulz, Oberampfrach, Wendelstein und Ammerndorf, als Pfarrer die von Neuhaus b. Höchstadt a. Aisch, Sommersdorf b. Ansbach und Windsheim inne, wo er am 12. Juli 1885 starb.

Dies die kurzen Daten seines Lebens. Mehr darüber zu sagen, verbieten die Raum- und Zeitverhältnisse, zumal wir ja auch einen ausführlichen Nachruf über ihn, nebst Verzeichnis seiner Arbeiten, aus der Feder seines Freundes Viktor R. von Tschusi zu Schmidhoffen besitzen. ${ }^{1}$ ) Es soll daher hier nur auf die Bedeutung Jäckels als Ornithologe eingegangen werden.

Zunächst durch seinen Vater, der eifriger Vogelliebhaber war, dann durch dịe Gebrüder Sturm angeregt, benützte J. jede freie Stunde zum Studium der Natur und zum Sammeln von Pflanzen und Tieren. Auch lernte er bald das Ausstopfen von Vögeln und Säugetieren, das er lange Jahre mit Geschick und künstlerischem Geschmack betrieb.

Als Jäckel im Jahre 1848 zum ersten Male mit einer wissenschaftlichen Arbeit an die Oeffentlichkeit trat, besafs er bereits eine eingehende Kenntnis nicht nur der Vogelwelt, sondern auch sämtlicher anderen Tierklassen sowie der Pflanzen. Sein scharfes $\mathrm{Ohr}$ rühmte sich, die beiden Baumläuferarten mit Sicherheit am Lockruf unterscheiden zu können, auch hatte J. auf Ausflügen bereits einen grofsen Teil Mittelfrankens durchstreift. Sein Ruf als Naturkenner war schon in weite Kreise gedrungen, von überallher bekam er Vögel und andere Tiere zur Bestimmung zugesandt und wurde um Rat und Auskunft in naturwissenschaftlichen Fragen angegangen. Selbstverständlich waren ihm auch alle Geheimnisse der Vogelliebhaberei und des Vogelfangs wohlbekannt.

$\mathrm{Zu}$ systematischen Studien hatte J., dem auf seinen entlegenen Wohnsitzen jedes Vergleichsmaterial fehlte, kaum Gelegenheit, weshalb wir auch über solche Fragen nur wenig in seinen Schriften finden, zumal auch die Systematik damals erst in ihren Anfängen steckte.

Seine Lebensaufgabe erblickte J. darin, die V er b r e it u n g der in Bayern vorkommenden Tierarten zu erforschen, ihr widmete er mit rastlosem Eifer jede Stunde seiner durch Amtsgeschäfte und auch Krankheit meist karg bemessenen freien Zeit. Aus eigener Anschauung kannte J. ganz Mittelfranken, den südlichen und westlichen Teil Oberfrankens, sowie grofse Teile von

1) 13. Jahresbericht des Naturhist. Vereins zu Passau 1883-85. Herr v. Tschusi hatte auch die grolse Liebenswürdigkeit, mir die Briefe J.'s an ihn zur Einsicht zu überlassen, wofür ihm auch an dieser Stelle herzlichst gedankt sei. 
Unterfrankeu und Schwaben, von Altbayern eigentlich nur München. Besonders ergebnisreich waren die in Neuhaus verbrachten Jahre, in dessen teichereicher Umgebung J. eine Menge der seltensten Sumpf- und Wasservögel als Durchzügler oder Brutvögel feststellen konnte. ${ }^{1}$ ) Aus den übrigen Teilen Bayerns brachte ihm eine ungeheuer ausgedehnte Korrespondenz mit wohl sämtlichen damaligen bayerischen Naturkennern, mit Jägern, Lehrern, Geistlichen und Aerzten eine Fülle des wertvollsten Materials, das aufs sorgfältigste geprüft und gesammelt wurde. J. verstand es aber auch, wie selten jemand, anzuregen, zu ermuntern, Irrtümer auf die zarteste, liebenswürdigste Weise zu berichtigen und den Einsender durch lobende Nennung seines Namens zu weiteren Beiträgen zu veranlassen. Alle vor J. ererschienenen faunistischen Arbeiten behandeln fast durchweg nur Teile Bayerns und enthalten, was die Verbreitung betrifft, meist nur spärliche und allgemeine Angaben, ganz abgesehen von groben Fehlern und Irrtümern. In seinem nachgelassenen, 1891 erschienenen Werke ,Systematische Uebersicht der Vögel Bayerns" konnte J. 312 für Bayern nachgewiesene Arten aufführen und damit sein Vaterland in die Reibe der ornithologisch am besten durchforschten Länder Deutschlands stellen. Er schuf die Grundlage, auf der weiterzubauen für seine Nachfolger eine wesentlich leichtere Aufgabe war. Besondere Aufmerksamkeit schenkte J. bei seinen Forschungen der Ausbreitung neueingewanderter Tierarten, wie Girlitz, Haubenlerche und Wachholderdrossel.

Ueber die B i o lo gi e unserer Vogelwelt verdanken wir J. manche wertvolle Mitteilung. J., der selbst ständig Singvögel, seltene Wintergäste, Sumpf-, Wasser- und Raubvögel hielt, notierte sofort jede ihm neuerscheinende Beobachtung, um sie mit dem von ihm hochverehrten "Naumann" zu vergleichen. Eingelieferte Vögel wurden auf das genaueste auf ihren Kropfund Mageninhalt, auf Schmarotzer und Eingeweidewürmer untersucht. Durch die mühevolle und sorgfältige Untersuchung von mehr als 10,000 Eulengewöllen hat sich J. ein bleibendes Verdienst um die Wertschätzung dieser lange verkannten Vogelklasse erworben. Abnormitäten wie Schnabelmifsbildungeu, deforme Fufsbildungen, Leucismus, Hahnenfedrigkeit usw. erfreuten sich der besonderen Aufmerksamkeit J.'s, der dafür wohldurchdachte und geistvolle Erklärungen gab.

Hervorhebung verdient $J$. auch als $H$ is $t$ or i $\mathrm{ke} \mathrm{r}$. In allen grölseren Arbeiten J.'s finden sich historische Angaben aus seltenen alten Werken und Zeitschriften oder alte Gemälde, Abbildungen und Stiche von Vögeln erwähnt. (Nur Zorns treffliche Petinotheologie scbeint ihm merkwürdigerweise unbekannt

1) Die Vogel des unteren Aisch-, Seebach u. Aurachgrundes. 6. Bericht der naturf. Gesellschaft zn Bamberg 1863. 
geblieben zu sein.) Auch wurden alte Akten, Jagdrechnungen, Schufs- nnd Fanglisten aufgesucht oder aus den verschiedensten Archiven Bayerns verschrieben, um über die früheren Vorkommnisse genaue Aufschlüsse zu erhalten, auch die gesamte Literatur, soweit zugänglich, benützt und zitiert.

Endlich sei J., der Freud und Leid und den Tageslauf des von ihm betreuten fränkischen Landvolkes aufs genaueste kannte, als $\mathrm{H}$ e i m a t f o r s c h e r genannt. J., der mit Stolz und Bewufstsein Bayer und Franke war, versäumt nie, Lokalbezeichnungen, Volksnamen und Volksglauben zu erwähnen und bieten seine Werke auch in dieser Hinsicht eine reiche, noch lange nicht ausgeschöpfte Fundgrube. Eine von J.'s Arbeiten behandelt ja speziell Volkssitte, Aberglauben und Volksmedizin in Franken. ${ }^{1}$ )

In gleicher Weise, wie über die Vogelwelt, hat J. über die Säugetiere gearbeitet und uns wertvolle, beute kaum mehr zu beschaffende Angaben über längst ausgerottete Raubtiere hinterlassen. Besondere Aufmerksamkeit widmete er der wenig bekannten Familie der Fledermäuse (und ihrer Nahrung), von denen er mehrere Arten für Bayern als neu nachwies. Wir besitzen von ihm auch eine Arbeit über die Fische Bayerns, ${ }^{2}$ ) in der er mehrere von ihm entdeckte Weifsfischbastarde beschreibt, und eine über die Kriechtiere und Lurche Bayerns ${ }^{8}$ ) mit vielen biologischen und historischen Angaben. J. besafs auch eine ausgezeichnete Kenntnis der Insekten und hat seinerzeit das Wiener Nachtpfauenauge in Windsheim eingebürgert. Mehrere seiner Arbeiten behandeln die Geschichte der Heuschreckenzüge in Bayern. J. war auch guter Kenner auf dem Gebiete der Conchylien, ebenso auf dem der Pflanzenwelt, die Pilze mit eingeschlossen; im Garten pflegte J. stets eine Menge schöner Blumen und seltener Blattpflanzen.

J., der uns mehr als 100 verschiedene Veröffentlichungen hinterlassen hat, war kein Vielschreiber im gewöhnlich gebrauchten Sinne dieses Wortes. Seine ganze Kraft vereinigte er auf das von ihm geplante grofse Werk über die Säugetiere, Vögel, Fische, Lurche und Kriechtiere Bayerns. Die vielen kleineren Aufsätze J.'s stellen gleichsam nur Antworten des bayerischen Heimatzoologen vor, in denen er irgendwo gelesene unrichtige Angaben über bayerische Vorkommnisse richtig stellt oder umgekehrt Beobachtungen in anderen Landesteilen durch solche aus Bayern bestätigt und ergänzt.

\footnotetext{
1) Abhandlungen der naturbist. Gesellschaft zu Nurnberg II. 1861. XVIII. 1864.

2) Correspondenzblatt des zool. miner. Vereins zu Regensburg XXV. 1865.
} 
Ueber J.'s Arbeiten wie auch über seine prächtigen, mit zierlicher Handschrift geschriebenen Briefe ist die Weihe klassischer Bildung ausgegossen. Sich von Allgemeinheiten fernhaltend, erfeuen sie immer wieder durch ihre klare, eigenartige und anschauliche Darstellungsweise, die sich nicht seiten $\mathrm{zu}$ warmer Begeisterung steigert. Ueberall spürt man in ihnen den Mann, der den Gegenstand, über den er schreibt, vollständig beherrscht und meist noch etwas Neues aus seiner eigenen reichen Erfahrung darüber zu +rzählen weils. Von Natur friedfertig, ist es immer die Sache, für die J. kämpft. Findet er aber seine mühevollen und gewissenhaften Beobachtungen in leichtsinniger oder ungerechter Weise angezweifelt, so scheut er sich auch nicht, in seine gewandte Feder gelegentlich die Lauge beifsenden Spottes oder heimatlicher Derbheit einflielsen zu lassen.

J.'s Arbeiten sind anfänglich im Korrespondenzblatt und in den Abhandiungen des zoologisch mineralogischen Vereins zu Regensburg erschienen, einer Gesellschaft die sich unter der Leitung Herrich-Schäfers die Erforschung der bayerischen Fauna zur Hauptaufgabe gestellt hatte. Später wurde von ihm die „Naumannia“, unser ,Journal für Ornithologie“ und der „Zoologische Garten" (jetzt „Zoologischer Beobachter") bevorzugt.

J.'s Verdienste fanden Ausdruck in der Ernennung zum korrespondierenden oder Ehrenmitglied zahlreicher wissenschaftlicher Vereine, wovon hier nur die Ernennung zum Mitgliede der kaiserlich Leopold. Carolin. Akademie der Naturforscher unter dem Beinamen „Fr. von P. Schrank“ erwähnt sei. Von seinen Windsheimer Mitbürgern, die den hochgewachsenen, äufserst liebenswürdigen Mann als treubesorgten Seelsorger und glänzenden Kanzelredner schätzten, hatten wohl die allerwenigsten eine Ahnung von seiner wissenschaftlichen Bedeutung, so still und schlicht, so gar nichts aus sich machend, lebte er unter ihnen.

Durch seine zahlreichen, gewissenhaften und gründlichen Forschungen hat sich J. selbst ein unvergängliches Denkmal gesetzt und sein Name wird unter den bayerischen Naturforschern stets in erster Reihe genannt werden. Mögen diese Zeilen dazu beitragen, die Erinnerung an ihn auch in Ornithologenkreisen wieder wachzurufen!

E. Gebhardt-Nürnberg. 


\section{$2 \mathrm{BHL}$ Biodiversity Heritage Library}

Gebhardt, Erwin. 1922. "Andreas Johannes Jäckel." Journal $f u$

r Ornithologie 70(1), 56-60. https://doi.org/10.1007/bf02540392.

View This Item Online: https://www.biodiversitylibrary.org/item/215787

DOI: https://doi.org/10.1007/bf02540392

Permalink: https://www.biodiversitylibrary.org/partpdf/210631

\section{Holding Institution}

Smithsonian Libraries

\section{Sponsored by}

Biodiversity Heritage Library

\section{Copyright \& Reuse}

Copyright Status: Not in copyright. The BHL knows of no copyright restrictions on this item.

This document was created from content at the Biodiversity Heritage Library, the world's largest open access digital library for biodiversity literature and archives. Visit BHL at https://www.biodiversitylibrary.org. 